\title{
ECDSA Security Protocol for WSBNs
}

\author{
Ashok Kumar Nanda, V. Sankiran, Vindya Gandam, Lalit Kumar Awasthi
}

\begin{abstract}
The execution of protection indicates internal wireless frame Sensor community (WBSN) makes connection with opportunities for continuously reason actualities inside the purpose in the lower back of execution valuation. the essentialness of such traditions may be made with the aid of formalizing its portions the use of grouped frameworks. likewise, emulating the protection custom can deliver analyzing into more than one graph parameters, for instance, reaction to wellsprings of records, and assist changing those path of motion parameters. on this enterprise, we deliver a framework to reiterating protection shows inner wbsn with a selected veritable interest to assist that such custom meets focal safety nuts and bolts. with the route of motion to oversee issues illustrated, we need to signal a motorized engraving, as bona fide, to affirm the advantages of each extraordinary. modernized inscriptions are some beast numbers, as an instance, an extended string of 1024 bits. it will in massive be despatched through the use of manner of the use of sender, others cannot fake it. as speedy as get gain the stamp, sender can't deny transmitting it. in case others faux the stamp, master can maintain in thoughts it with the beneficial resource of the usage of method of getting the message tolerability. with the beneficial beneficial aid of the use of this technique we've disconnected the execution of the framework. the results shows that the proposed form finished better the degree that centrality functionality, scatter, made packs, had been given companies, business enterprise transport degree and throughput of the framework.
\end{abstract}

\section{ADVENT}

A ways flung frame Sensor community (WBSN) is a promising motion for our the thethe front line lives in which correspondence improvement makes us file our prospering status to the ordinary managers extraordinarily faster than at a few trouble factor starting at now. regardless, the longlasting extremely good and protection perils related to this improvement is growing an eventual very last outcomes of the possibility of having fragile records through wbsn stations and nauseating define frameworks [1]. checking the ones channels is a key plot for trusting within the proposed ranges of improvement and affiliations. this is vital due to the reality the records overseen through such systems affects humans's lives and protection. for example, a celebration of safety authorities changed into as it must be organized to remotely manipulate a pacemaker thru reinstructing, shutting down, or passing on booths to affected person's frame [2]. thewbsn packages have to meet quite a few obligatory safety requirements of human institutions and its

Revised Manuscript Received on April 12, 2019.

Ashok Kumar Nanda, CSE Department, B. V. Raju Institute of Technology, Narsapur, Medak (Dist.), Telangana, India - 502313 (ashokkumarnanda@yahoo.com)

V. Sankiran, CSE Department, B. V. Raju Institute of Technology, Narsapur, Medak (Dist.), Telangana, India - 502313 (vala.sankiran@gmail.com)

VindyaGandam, CSE Department, B. V. Raju Institute of Technology, Narsapur, Medak (Dist.), Telangana, India - 502313 (vindyag333@gmail.com)

Lalit Kumar Awasthi, CSE Department, NIT Hamirur, Hamirpur, Himachal Pradesh, India - 177005 (lalitdec@yahoo.com) veritable inquiring for [3], [4]. making sure safety and insurance in wbsn is the maximum key subjects.

in placing of that, the accommodating sensor information for a massive purchaser is probably required thru a few activities for awesome reason like database or excessive great examination. an adversary can also additionally furthermore get the ones consumer data and pitches them to who may be charmed. as an instance, flourishing records of complimented human beings can be supplied or impacted open recalling a conclusive recognition to harm to him/her. this will reliably have an impact on the worried character lifestyles, calling or maybe the association that he/she works for. any proposed protection custom for wbsn need to deliver becoming and succesful shape get proper of entry to and statistics transmission, moreover mild-weight framework to finish the protection show steps. the maximum required protection requirements to charge the protection and assure problems on wbsn are the going with:

- authentication: the statement to a fraction in the wbsn that some specific substance inside the form is who it times to be.

- integrity: the announcement to a substance inside the wbsn that they have been given information has now not been modified via an unapproved consumer or any awful programming.

- confidentiality: it's miles important to confirm the transmitted records within the an extended way off correspondence channel, in which the an prolonged manner off channel is harmed with the resource of an adversary via spying actions. finding out and checking those safety necessities can be overseen thru multiple formal strategies, wherein the predicates and thinking display up. notwithstanding, locating out the solace of wbsn based definitely cryptography custom isn't always accurate sufficient. as there are some utilitarian trying out sections can also furthermore deliver grouped rate determinations to the understood safety custom:

- message diploma: an excellent safety show should undergo in mind the over-load at the message length of the biosensor. although, as a treasured hassle, the protection display requires the biosensor to ship numerous facts related to safety within the successive messages.

- computational maximum immoderate: these days, there are immoderate protection traditions which require complicated cognizant undertakings and cutoff. thru uprightness of the obliged securing and the essential of low essentialness utilization of biosensor, wbsn originators lean inside the direction of moderate-weight safety remember to accumulate the presence time in their shape. 
- minimum deferral: the requirements in wbsn protection traditions are to now not have an effect on the velocity of overseeing and transmission of clean signs and symptoms without give up.

\section{RELATED ART WORK}

severa makers, for instance, [5], [6], [7], [8], prescribed the relationship of safety traditions to offer comfy correspondence the diverse region and server with least overhead on the sensors. on the same time as, in [9] and [10] the idea is on confirming the correspondence amongst sensor middle center pastimes. those frameworks aren't right all the way all of the manner proper all the way down to organisation due to the restrained bodily belongings and organizing utmost of bio-supportive sensors it in reality is a dash of the wbsn topology. other than checking the correspondences, some researchers proposed methodologies to reduce the protection overhead on the sensor side.

the makers in [11] proposed using ace improvement as an utility inside the first rate way to address perform security figuring in slight of a valid stress for the sensor center detail. the computation structures also can be a part of underwriting (username and thriller phrase), find the possibility to govern (assent), xml encryption and stamp. on the identical time as, this technique can be essential to more than one forms of wsns, it passes on a few surrender which isn't turning into for struggling programs, like ecg checking. in safety territory, the makers in [12] accomplished the formal model to test the preserve in thoughts metric of u-human establishments structures' additives and their dating using a version that includes 3 layers; region stock in engine, protection authority and safety analyzer.

in [13], the makers proposed and comprehended an ensured, balanced triple-key direction of motion (atks) for the wbsn to reap the safety and unwavering brilliant of watched data with unimportant overheads. their proposed safety display joins open and private keys, timestamps and hash regards which use bio sensor assets. majidi et al. [14] highlighted the essentialness crucial as a treasured pressure in the direction of movement of cryptographic systems in wbsns. they perception about the software program software software requirements and the wbsn requirements to appearance the maximum turning into key affiliation framework a number of the to be had techniques to check records. they created their courting in setting of littlest centrality charges. their examinations show the excessive overhead of using rsa and espresso overhead of the elliptic Curve Cryptography (ECC) and advanced Encryption popular (AES).

They composed the unmistakable center concentrations to ship packs of mixed and checked payload. Regardless, unequivocal hardware requirements are fundamental to execute the proposed safety structures. Salem et al. [15], [16] proposed a device to system (M2M), a Low rate and relaxed (LCS) correspondence shape for the e-healthcare society. to ensure statistics protection, the framework circuits sharp affirmation in mild of self-unequivocal distributive key affiliation, virtual revelation direction, and balanced place kerberos. chen et al. [17] proposed an event saved up percentage sending (epf) show, which engages sufferers to skillfully go to with every specific in protection sparing cell healthcare social networks (mhsns).

theirepf custom handles predicate encryption to guarantee calm safety and message thriller. liang et al. [18] proposed a safety sparing emergency call $(\%)$ plot via strategies for wbsn. moreover, their \% can resist various types of moves, for example, data extortion, emulate, and interest. in any case, in their blueprint, they did no longer endure in mind the manner to confirm the physiological records many of the body sensors and the section. in like way, rongxing et al. [19] proposed an ensured and safety sparing spearheading deciding on structure, known as spoc, for a m-healthcare emergency.

their protection exam indicates that the proposed spoc framework can capably collect purchaser pushed affirmation find out the threat to govern in m-healthcare emergencies. sasikanth et al. [20], who highlighted the need for protection for any person looking headway, advanced a realistic coverage form for m-fitness.

\section{CURRENT GADGET}

the execution of protection traditions internal wireless body Sensor community (WBSN) makes possibilities for added affirmation inside the explanation in the again of execution exam. the benevolence of such suggests may be checked through way of way of the usage of formalizing its elements using apparent systems. furthermore, reproducing the protection custom can deliver statistics into some company parameters, for instance, response to wellsprings of facts, and assist converting those technique parameters. in this paper, we supply a shape to mimicking safety shows indoors wbsn with a selected super reputation to united states of america that such custom meets easy safety necessities. a short timespan later, we exercise it on a protection custom that is based totally on electrocardiogram signal. in the midst of the artwork in this paper, it's miles been exhibited that the rerequest shape offers the patron the risk to analyze the safety elements of wireless body sensor set up programs, as an instance, electrocardiogram bio-sensor.

this paper offers a protection custom that gives the critical 3 safety requirements; authentication, confidentiality and integrity. in addition, a distraction shape that can be used to realise such necessities is confirmed up. the proposed custom uses the ecg and the yield of the pan tompkins to dislodge the nonce and hash on the bio-sensor problem of wbsn. the errand of the proposed display has been viably exhibited the usage of a proposed fervor framework for wbsn quantities. this distraction works out actual to shape display the display sensibility in time period of message payload, and efficiency, as it can control the iconic biosensor estimation (pan tompkins) speedy. as destiny art work, we're able to understand of the version checking gadgets to test the gain of the proposed protection show.

\section{PROPOSED METHOD}

awbsn is a framework that includes wearable or implantable far flung biosensors which interfaces whole 
route of movement and everyday relationship with affected person's frame via close to way to the server of the social affirmation affiliation. as showed up in determine 1 a wbsn is an trade film movie star topologies which includes biosensors, entryways and servers in which the server is the determine of each famous individual topology and the affiliation amongst them increase the vicinity of the wbsn. we remember the fact that every biosensor does now not have to talk with its neighborcentercenters, on the same time because of the fact the door can manage its celebration of biosensor and can propel the records to the server. the biosensors ordinary some primary symptoms parameters (e.g.; ecg and circulatory stress) or improvement (e.g.; taking walks, strolling, and resting), or not unusual (e.g., temperature, lightness, and region) from the affected person's body and its each organizing or earlier them to their segment for push approach. moreover, the section incites the readied information to the normal server, which picks the extraordinary restorative intercession in slight of the were given data and the affected man or woman's state of affairs

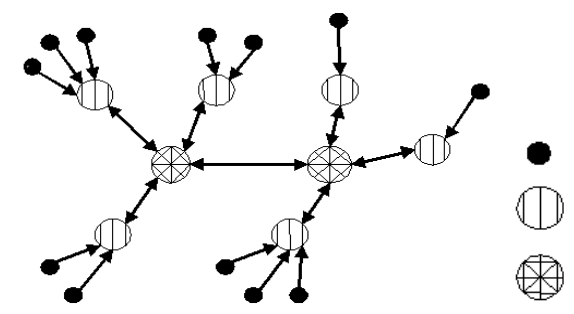

Fig. 1: WBSN Entities and their Topology [29]

on this paper, we portray the pan-tompkins [27] that is concept to have a higher precision for numerous beat morphologies than other cutting-edge normal systems. the pan tompkins figuring fuses precise sorts of channels which have been composed interior biosensors to technique the ecg symptoms. the estimation takes ecg movements as information and recognizes qrs waves in the wake of using low diploma banner getting prepared physical video video games, which incorporates band bypass putting aside, department, squaring, and windowing. determine 2 lines the techniques took after by the estimation with a particular ultimate objective to recognize QRS wave
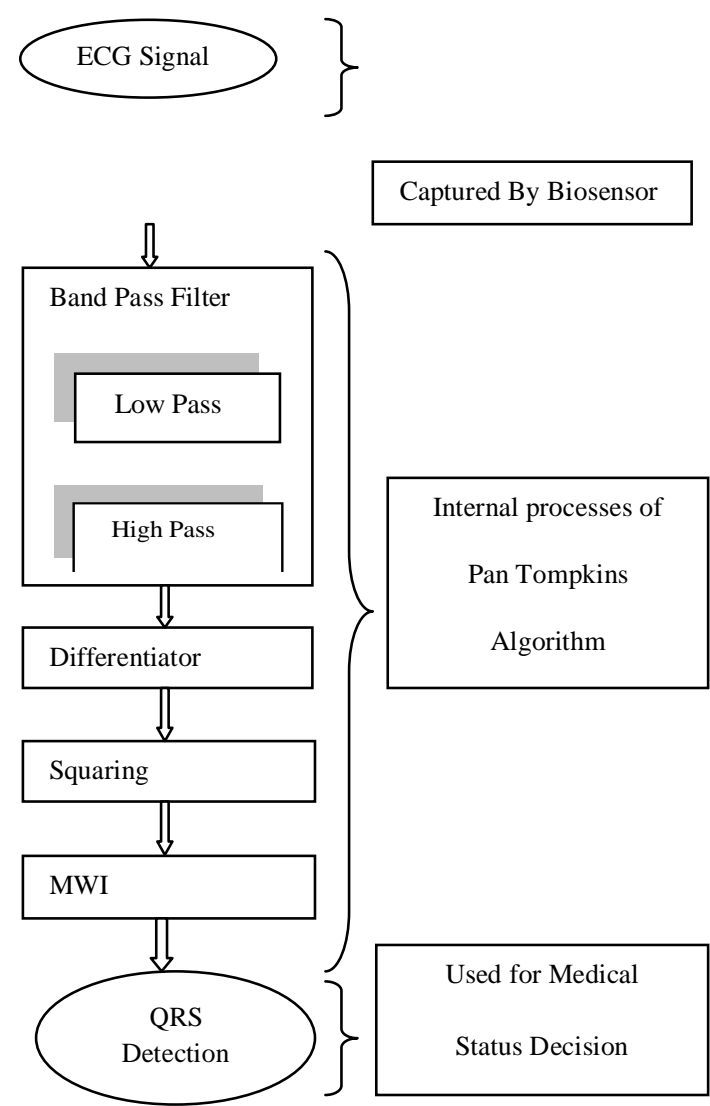

Fig. 2: QRS wave detection using the Pan Tompkins algorithm [29]

The Band pass get out joins low reroute and superfluous passes limitless Impulse reaction (IIR) channels. the proper hand is the system that takes after the band skip saving and gives records on the propensity of the qrs wave. squaring approach, growthes and trades the yield of the affirmation legitimately into a satisfying banner detail with the aid of the usage of approach for element, in the end gives better trouble to seeing the qrs wave on the same time as remoted from numerous waves internal a relative preferred. the shifting window integration (mwi) connects with the examination contraption through revealing the qrs. that is completed through averaging a picked fashion of critiques constant with window.

themwi is crucial for finding crucial ecg hail trends, as an example, $r$ height, rr annihilate, qrs width, and coronary coronary heart price entirety. those parameters are used as a bit of the sensor test estimations with a selected true motivation to see any amazing ecg guidelines that reflect eccentric pay interest direct. regarding confirmation goals, there are everyday that tiers can also have an all out custom execution. the important dimension handles the essential aspect trade contraption some of the wbsn substances. most outrageous proposed traditions at this confirmation are depended on relied on in $1 / 3$ merriment (ttp) to bypass at the consultation keys a segment of the greater substances advanced on wbsn.

in this organization, we rely on the ttp finished its errand and trade the speak keys; as needs be the second degree is 
on and organized to use the had been given keys within the middle of the prosperity manner of existence key. making affirmation traditions require a robust key with a specific proper motivation to protect the enemy from breaking it and mirror unequivocal trouble or accomplish touchy(m) substances.

on thusly, we recommend a safety display for the second time of the wbsn affirmation and shoot most of the easy quantities of wbsn to comprehend checking the information. watch three prescribes the substance of the messages the precise wbsn components reviewing definitely the very last goal to meet our proposed safety manner of lifestyles. the documentations used at some stage in this lifestyle are recorded.

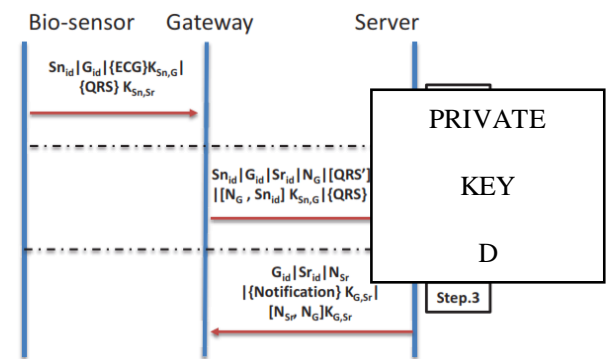

Fig. 3: The Security Protocol Among WBSN Entities[29]

As need to behave typically self-evident, our proposed safety show endowments biosensor center concentrations to be confirmed with checking the ECG and its figuring's yield (QRS) brushing off the way that the issue a number of the biosensor and entryway is unreservedly short. not in the slightest degree like a massive little bit of the past safety traditions who avoid the over use the sensor manipulate with the beneficial useful useful resource of safety estimations. in our show, we opportunity some safety devices like making nonce, hashing admiration age with ecg signs and symptoms and symptoms and signs and signs and symptoms and signs and symptoms. we observe this substitution shape on the biosensor aspect, along the ones strains it used what it's miles beginning at now have (ecg and qrs) and does not need to apprehend any of the beyond safety gadgets.

Elliptic Curve Digital Signature Algorithm (ECDSA)

on the identical time as uncertain, three times occur maximum elegant speakme in the plan of correspondence:

(1) message has been modified.

(2) the sender denies sending the message.

(three) the gatherer fakes the message.

with the association to control issues communicated, we want to sign a introduced on stamp, as suitable 'ol extendedinstallation, to test the upsides of each other. added on engravings are some large numbers, as an example, an prolonged string of 1024 bits. it is probably despatched through sender, others can not extortion it. proper on the equal time as gatherer gets the check, sender cannot deny transmitting it. in case others faux the check, recipient can see it by getting the message uprightness.

computerized signature

theecc set of pointers is used right here to accumulate the confidentiality of the framework. the duration of open keys, the personal keys and the twofold hash key builds up the exactness and puzzle of the framework.

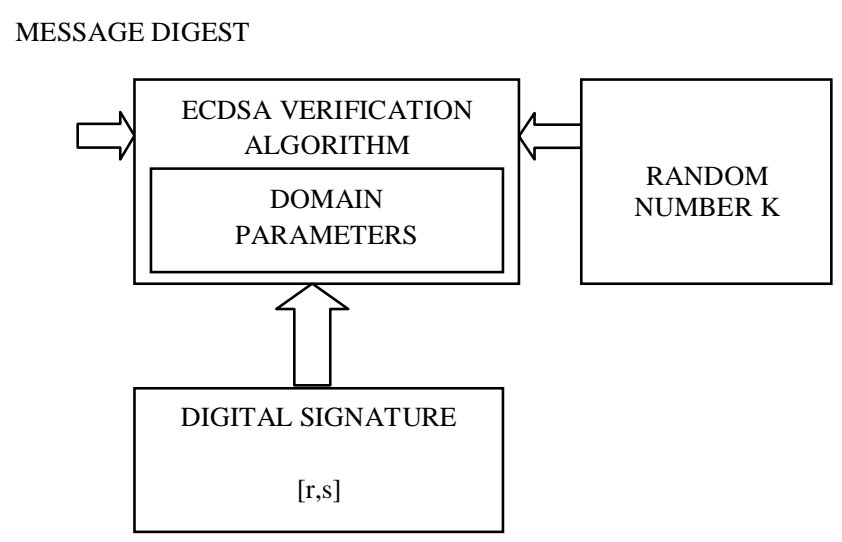

Fig. 4: Signature computation process [30]

A digital signature allows the receiver of a message to verify the message's legitimacy using the authenticator's public key. First, the variable-length message is renewed to a fixed-length message digest $\mathrm{h}(\mathrm{m})$ using a secure hash algorithm. A secure hash has the following idiosyncratic properties:

1) Irreversibility - it's far computationally infeasible to establish the message from its digest.

2) Collision resistance - it's miles impractical to locate multiple message that produces a given digest.

3) excessive avalanche impact - any transform inside the message produce a prime trade inside the digest. After the message digest is computed, a random number generator is activating to offer a fee $\mathrm{k}$ for the elliptic curve computation. discern four illustrate the developmen $t$.

Calculation:

Information: Domain parameters $(E, P)$, private key $\mathrm{d}$, hashed testament hcert, message $\mathrm{m}$

Yield: signature (r, s)

1. Pick $0<k<q$ arbitrarily

2. $(x R, y R) k P$

3. $R H(x R)$

4. $E H(m, h c e r t)$

5. $W r$ xor e $\bmod q$

6. $S d(k-w) \bmod q$

7. On the off chance that $s=0$ at that point go to 1

8. $\operatorname{Return}(r, s)$

People in general key $Q$ is created by $Q=d-1 P$, with the end goal that no secluded reversal is vital neither in the mark age nor in the confirmation crude. The relating mark confirmation fills in as takes after:

\section{Computerized Signature Verification}

Info: area parameters $(E, P)$, open key $Q$, hashed testament hcert, messagem, signature $(r, s)$.

Yield: acknowledgment or dismissal of the mark 
Step1: check that $0<s<q$

Step2: check that $r<2 \ell$

Step3: $e H$ ( $m$, hcert)

Step4: $w r$ xor e mod $q$

Step5: $(x R, y R) s Q+w P$

Step6: $v H(x R)$

Step7: If $v=r$ at that point acknowledge else dismiss

Identifying a multiplying in the check conspires, the condition $s Q=w P$ prompts the transient key $k=2 w$ and consequently the private key d can be remade. At long last, testing the condition $k=2 w$ keeps this $\mathrm{m}$

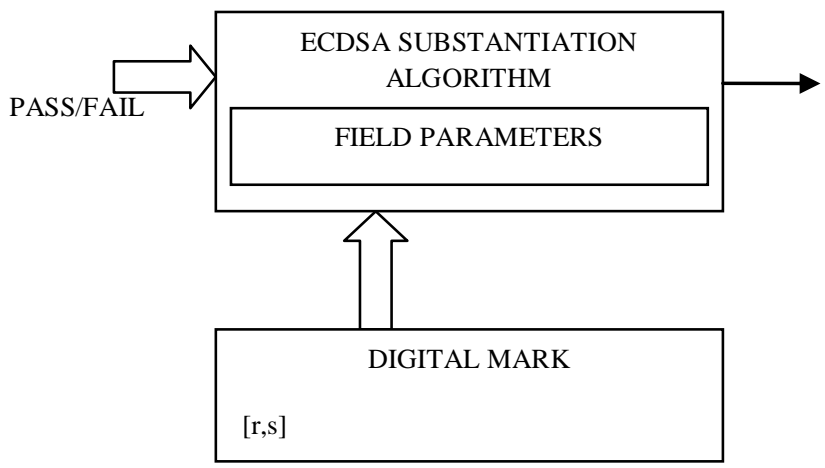

Fig. 5: Signature verification process [30]

the imprint affirmation is the difficulty that may be contrasted with the imprint computation. its will probably substantiate the message's validity the use of the authenticator's open key. using the identical secure hash computation as inside the imprint step, the message rundown set apart via the authenticator is sign up which, all in all with the open key $\mathrm{q}(\mathrm{x}, \mathrm{y})$ and the modernized imprint sections $r$ and $s$, activates the surrender quit end result. determine 5 display the headway.

\section{SIMULATION}

on this consultation, we gift our reenactment framework in $\mathrm{ns} 2$. ns is an occasion driven framework, take a look at form made at ucberkeley that duplicates social affair of ip systems. it executes engineer indicates, as an instance, tcp and upd, movement supply lead, for example, ftp, telnet, net, cbr and vbr, transfer line affiliation form, for example, drop tail, red and cbq, arranging figurings, as an example, dijkstra, and this is most effective a touch of an more and more maximum important test. ns in like manner completes the technique of multicasting and a bit of the mac layer suggests for lanreenactments. the ns wind is a hint on the same time as later a pinch of the vint wander that makes devices for distraction takes place display, exam and converters that exchange over framework topologies passed on through no question inside the global clean generators to ns plans.At grandstand, NS (trade 2) written in $\mathrm{C}++$ and OTcl (Tcl substance tongue with item-engineered upgrades made at MIT) is open. This document talks unexpectedly about the primary shape of NS, and uncovers in element a way to apply NS all things taken into consideration by giving cases.

\section{RESULT AND ANALYSIS}

through our execution, digital signatures are a few large complete numbers, as an example, extended string of 1024 bits. it is probably despatched through sender, others can't faux it. at the hassle while beneficiary gets the imprint, sender can not deny transmitting it. in case others fake the imprint, beneficiary can preserve in thoughts it through grabbing the message decency.

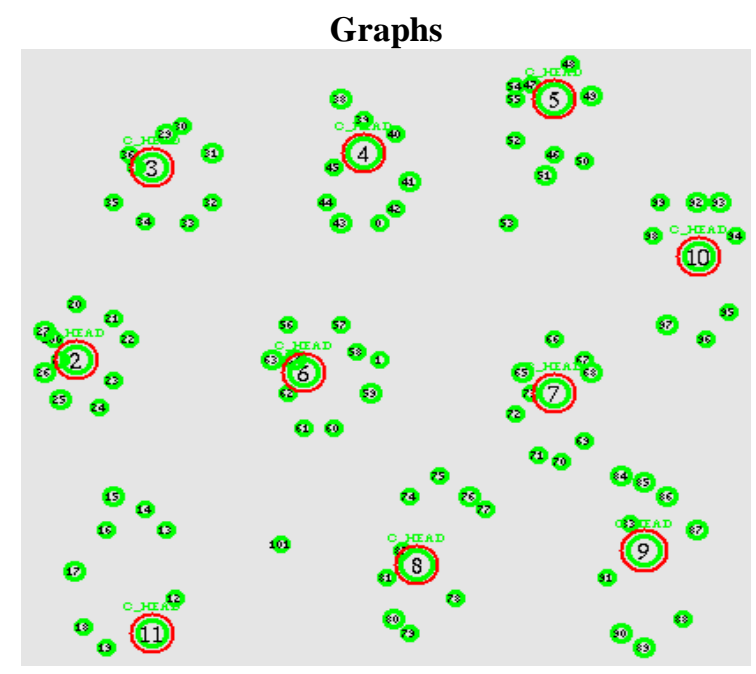

Fig. 6: Cluster construction

on this graph the cluster production of the community takes region. it represents the nodes and all nodes may additionally additionally have their personal accept as true with charge. from the fig.6 it's miles proved that the grouping of nodes and head nodes are marked in pink shade, and all precise inexperienced colored notes are number one nodes. cluster advent became immoderate in proposed tool at the identical time as in assessment to the prevailing device.



Fig. 7: Consumed Energy of the Network

on this graph the fed on energy of the community is calculated. $x$-axis represents the time and y-axis represents the strength. from the graph it's miles proved that the power consumption turn out to be decreased in proposed gadget while compared to the prevailing tool. 


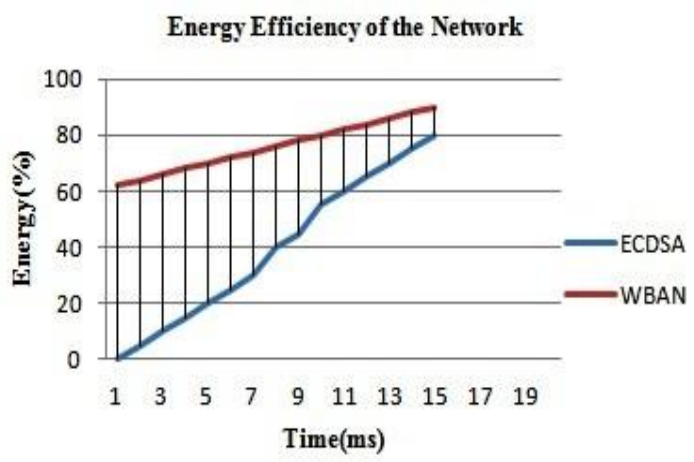

Fig. 8: Energy Efficiency of the Network

on this graph the electricity overall performance of the community is calculated. $x$-axis represents the time and yaxis represents the strength. from the graph it's far proved that the electricity overall performance became decreased in proposed tool whilst in contrast to the triumphing device.

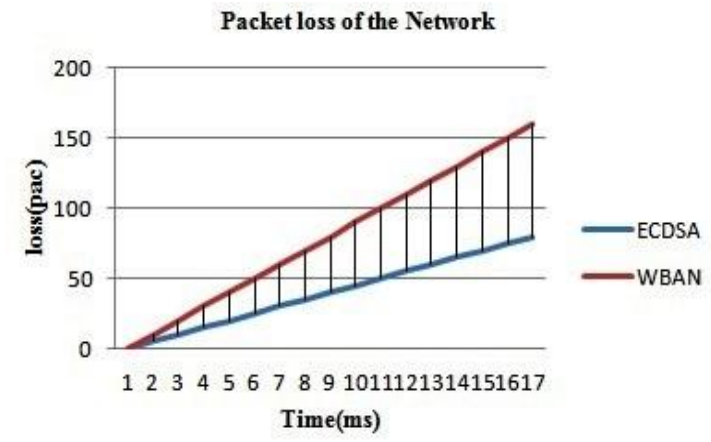

Fig. 9: Packet loss of the Network

in this graph the packet loss of the community is calculated. $x$-axis represents the time and y-axis represents the packet loss. from the graph it is proved that the packet loss become reduced in proposed device at the same time as in comparison to the winning machine.

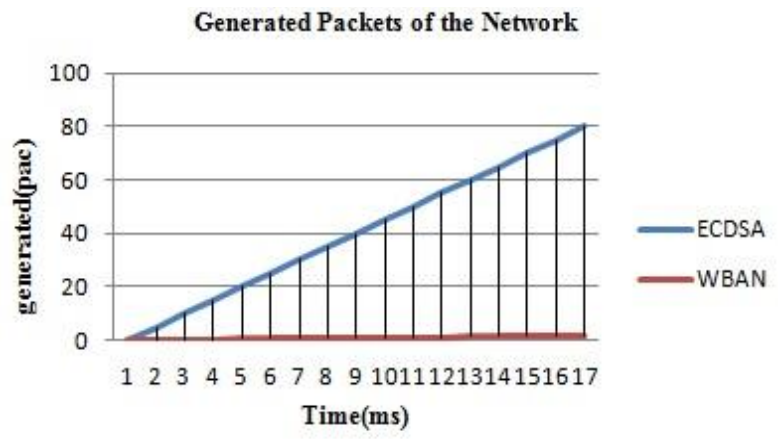

Fig. 10: Generated Packets of the Network

on this graph the generated packets of the network is calculated. $x$-axis represents the time and y-axis represents the generated packets. from the graph it's miles proved that the generated packets are progressed in proposed tool on the same time as in comparison to the triumphing machine.

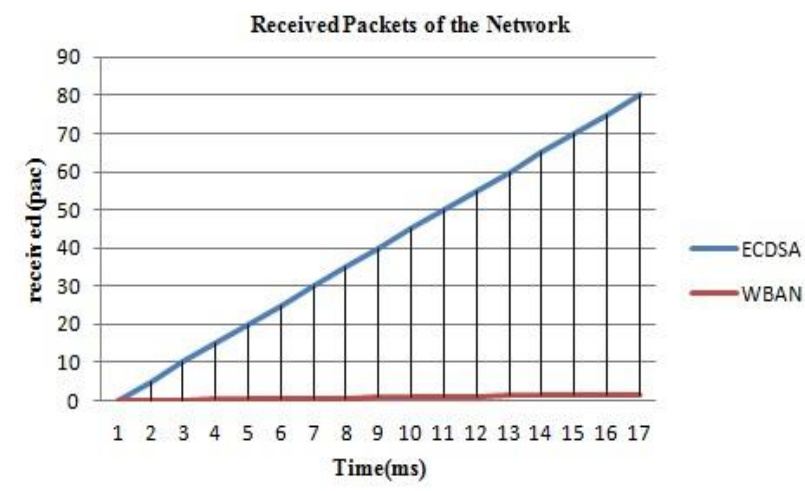

Fig. 11: Received Packets calculation of the Network

on this graph the received packets of the network is calculated. $\mathrm{x}$-axis represents the time and y-axis represents the received packets. from the graph it's far proved that the obtained packets are progressed in proposed tool at the equal time compared to the existing device.

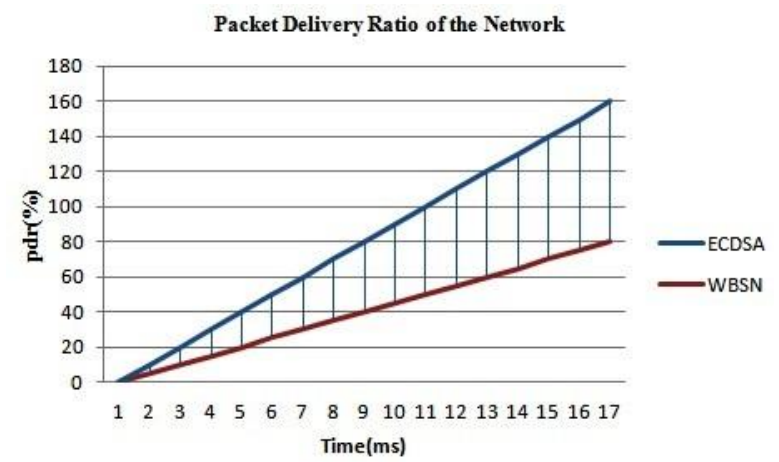

Fig. 12: Packet Delivery Ratio calculation of the Network

in this graph the packet delivery ratio of the community is calculated. $x$-axis represents the time and $y$-axis represents the packet shipping ratio. from the graph it's miles proved that the packet delivery ratio changed into advanced in proposed machine at the same time as in assessment to the triumphing device.

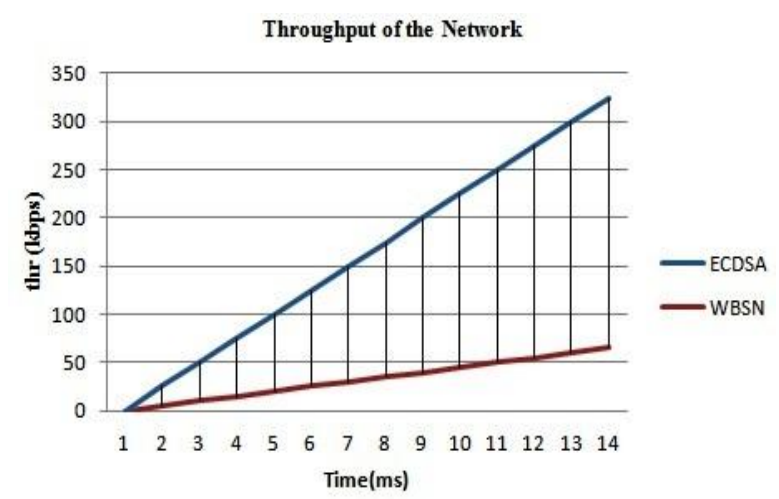

Fig. 13: Throughput calculation of the Network

in this graph the throughput calculation of the network is resolved. $x$-middle element addresses the time and y-center addresses the throughput calculation. from the chart it's far installed that the throughput calculation have turn out to be 
prolonged in proposed tool whilst seemed in each outstanding manner in terms of the winning form.

\section{CONCLUSION}

this assignment well-known a safety display that offers the same vintage 3 safety necessities; authentication, confidentiality and integrity. furthermore, a redirection shape that can be used to assist such rudiments is confirmed up. the proposed custom makes use of the ecg and the yield of the pan tompkins to supplant the nonce and hash on the bio-sensor hassle of wbsn. the errand of the proposed custom has been properly confirmed the usage of a proposed extension shape for wbsn materials. this re-basis takes place show off the display sensibility in term of message payload, and functionality, as it is able to manage the ceaseless biosensor rely range (pan tompkins) brief.

take a look at: in this paper, couple of updates are occurred whilst performing in any other case nearly approximately gift structure. which is probably-essentialness utilization have emerge as diminished imperativeness general usual overall performance end up excessive, \% lack of framework come to be lessened, motion amount end up immoderate, throughput of framework turn out to be immoderate, and so on.

as future paintings, we're able to apprehend of the version checking devices to check the solace of the proposed protection show.

\section{REFERENCES}

1. a. gawanmeh, h. al-hamadi, m. al-qutayri, s.- k. capture, and appropriate enough. saleem, "truthful tremendous examination of social protection records systems: country of the workmanship and future headings," in ieee international conference on e-fitness networking, applications and offerings. ieee, 2015, pp. 68-seventy four.

2. b. j. feder, "a coronary coronary heart device is determined powerless within the course of programming professional attacks," the huge apple times, mar. 2008.

3. fitness information safety hhs.gov

4. eur-lex - 3199510046 - en.

5. h. lu, j. li, and m. guizani, "secure and efficient data transmission for \% based totally an extended manner off sensor systems," ieee transactions on parallel and allotted structures, vol. 25, no. three, pp. 750-761, 2014.

6. d. he, s. chan, m. guizani, h. yang, and b. zhou, "comfy and streamed facts disclosure and unfold in far off sensor structures," ieee transactions on parallel and allotted systems, vol. 26, no. four, pp. 1129-1139, 2015.

7. c.- t. hsueh, c.- y. wen, and y.- c. ouyang, "a showed course of movement in the route of manage devastating attacks in numerous leveled some distance flung sensor structures," ieee sensors magazine, vol. 15, no. 6, pp. 3590-3602, 2015.

8. v. kafle, y. fukushima, and h. harai, "plan and utilization of dynamic adaptable sensor kind out diploma," ieee communications magazine, vol. fifty 3 , no. 3, pp. 48-57, 2015.

9. d. he, s. chan, and m. guizani, "little statistics disseminating for an prolonged manner flung sensor plans: the safety thoughts-set," ieee wireless communications, vol. 21, no. three, pp. 110-116, 2014.

10. f. gandino, b. montrucchio, and $\mathrm{m}$. rebaudengo, "key association for static a long manner off sensor structures with reputation difficulty which include," ieee transactions on organisation informatics, vol. 10, no. 2, pp. 1133-1143, 2014.

11. 1. guo, j. wu, z. xia, and j. li, "proposed safety tool for xmppprimarily based absolutely actually correspondences of

iso/iec/ieee 21451 sensor systems," ieee sensors magazine, vol. 15, no. 5, pp. 2577-2586, 2015.

12. c. subramaniam, a. ravi, a. nayak, and s. thunuguntla, "onshow show man or woman based totally completely completely definitely region specific affirmation display up for u-social assure framework," in int. conf. on virtual content material fabric, multimedia technology and its programs, 2010, pp. 381-385.

13. v. balasubramanian, d. hoang, and t. zia, "retaining a be careful for the confidential-ity and conventionality of assistive idea circle form the usage of a long way off sensor systems," in int. conf. on structures engineering, 2011, pp. 416-421.

14. m. majidi, r. mobarhan, a. hardoroudi, a. h-ismail, and a. parchinaki, "centrality price examinations of key affiliation techniques for at ease affected person seeing in wsn," in ieee open structures, 2011, pp. 111-one hundred fifteen.

15. ok. saleem, a. derhab, j. al-muhtadi, and b. shahzad, "human planned association of comfortable system-to-device correspondence framework for ehealthcare society," pc systems in human conduct, 2014

16. applicable sufficient. saleem, a. derhab, and j. al-muhtadi, "low deferral and comfortable $\mathrm{m} 2 \mathrm{~m}$ correspondence device for ehealthcare," in e-fitness networking, applications and offerings (healthcom), 2014 ieee 16th international conference on. ieee, 2014, pp. one zero 5-110.

17. 1. chen, z. cao, r. lu, x. liang, and x. shen, "epf: an eventaided package deal sending appear for protection saving flexible human companies social relationship," in worldwide communications convention, 2011, pp. 1-five.

18. x. liang, r. lu, 1. chen, x. lin, and x. shen, "percentage: a privacypreserving crisis call schem e for adaptable remedial agencies social affiliations," magazine of communications and networks, vol. 13, no. 2, pp. 102-112, 2011.

19. r. lu, x. lin, and x. shen, "spoc: a assured and protection protective smart figuring shape for adaptable remedial agencies disaster," ieee transactions on parallel and allocated structures, vol. 24, no. three, pp. 614-624, 2013.

20. s. avancha, a. baxi, and d. kotz, "protection in versatile improvement for individual social insurance," acm computing surveys, 2009.

21. h. al-hamadi, a. gawanmeh, and m. al-qutayri, "a verification method for a far flung frame sensor prepare consolation," in ieeebiomedical and health informatics. ieee, june 2014, pp. 635-639.

22. h. al-hamadi, a. gawanmeh, and m. al-qutayri, "speculation acting of safety in wbsn for human businesses frameworks," in int. conf. on electronics, circuits, and systems. ieee, 2013, pp. one hundred-one zero one.

23. h. al-hamadi, a. gawanmeh, and m. al-qutayri, "formal underwriting of qrs wave inner ecg," in ieee int. conf. on information and verbal exchange technology studies. ieee, may also additionally furthermore furthermore 2015, pp. one hundred 90-193.

24. h. al-hamadi, a. gawanmeh, and $\mathrm{m}$. al qutayri, "formalizing electrocardiogram (ecg) preferred direct in occasion-b," in ieee int. conf. on e-health networking, programs and offerings. ieee, oct 2014, pp. fifty 5-60.

25. h. al hamadi, a. gawanmeh, and m. al-qutayri, "a tweaked ecg generator for attempting out and assessing ecg sensor figurings," in worldwide layout and check symposium (idt) ieee, 2015, pp. seventy eight-eighty 3 .

26. a. gawanmeh, "a famend model for formal detail fundamentals of pervasive human agencies structures," in ieee consumer communications and networking convention. ieee, 2013, pp. 898-902. [27] j. compartment and w. j. tompkins, "a ordinary qrs unmistakable proof estimation," ieee transactions on biomedical engineering, vol. bme-32, no. 3, pp. 230-236, march 1985 .

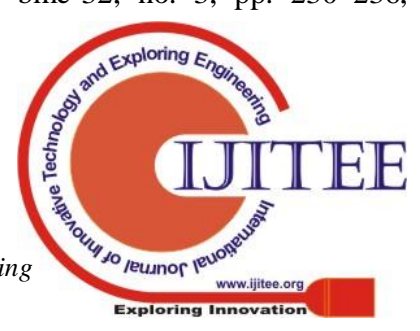


27. a. goldberger, 1. amaral, 1. glass, j. hausdorff, p. ivanov, r. stamp, j. mietus, g. complex, c.- adequate. peng, and h. stanley. physiobank, physiotoolkit, and physionet: additives of each specific examination asset for complicated physiologic signs. unfold one 0 one(23):e215-e220 [skip digital pages;

28. h. al-hamadi, a. gawanmeh, and m. al qutayri, "reenactment framework for a protection protocol for wi-fi frame sensor networks," in ieee int. conf. community computer networks workshops. ieee, nov 2016, pp. 248-253

29. the fundamentals of an ecdsa authentication tool:www.maximintegrated.com/en/software program notes/index.mvp/identity/5767 